\title{
Analytic image concept combined to SENSE reconstruction
}

\author{
Josiane Yankam Njiwa • Christof Baltes • \\ Markus Rudin
}

Received: 4 June 2010 / Revised: 1 June 2011 / Accepted: 13 July 2011 / Published online: 11 August 2011

(C) The Author(s) 2011. This article is published with open access at Springerlink.com

\begin{abstract}
Object Two approaches of reconstructing undersampled partial $k$-space data, acquired with multiple coils are compared: homodyne detection combined with SENSE (HM_SENSE) and analytic image reconstruction combined with SENSE (AI_SENSE). The latter overcomes limitations of HM_ SENSE by considering aliased images as analytic thus avoiding the need for phase correction required for HM_SENSE.

Materials and methods In vivo imaging experiments were carried out in male Lewis rats using both gradient echo and spin echo sequences. Accelerated images obtained by using the various reconstruction algorithms were compared to fully sampled reference images both qualitatively and quantitatively.

Results For the various sampling patterns evaluated, both HM_SENSE and AI_SENSE were found to yield robust image reconstruction with small deviations from the reference image. Even for high acceleration factors AI_SENSE still provided useful results and was found superior compared to HM_SENSE.

Conclusion Combination of partial $k$-space sampling and parallel image acquisition allows for further acceleration of data acquisition as compared to each method alone. Image reconstruction from undersampled data sets using the AI_SENSE algorithm was found to considerably reduce reconstruction errors and artifacts observed for HM_SENSE reconstruction caused by errors in phase estimation.
\end{abstract}

J. Yankam Njiwa $(\bowtie) \cdot C$. Baltes · M. Rudin

Institute for Biomedical Engineering, University and ETH Zurich, AIC-ETH, HIT E22, Wolfgang Pauli-Strasse 27,

8093 Zurich, Switzerland

e-mail: joad24@yahoo.com

M. Rudin

Institute of Pharmacology and Toxicology, University of Zurich,

Zurich, Switzerland
Keywords pMRI $\cdot$ SENSE - Analytic image $\cdot$ Partial $k$-space reconstruction $\cdot$ MRI $\cdot$ Constrained reconstruction

\section{Introduction}

Parallel magnetic resonance imaging (pMRI) is a fast imaging approach that uses an array of RF receiver coils to simultaneously acquire multiple sets of subsampled $k$-space data. The advent of pMRI has opened a door for collecting data using less time. Over the years, a number of strategies have been proposed for reconstructing full resolution and aliased-free images from the acquired undersampled $k$-space data. Reconstruction in image domain is based on merging images reconstructed from each coil element with reduced FOV using knowledge of individual coil sensitivities. The inverse problem that recovers spatial information from a set of radio frequency coils at different spatial positions can be solved in either $k$-space domain, it is assumed that spatial harmonics of the phase encoding gradients can be omitted and estimated by linear combination of coil sensitivities then missing $k$-space lines are explicitly computed before Fourier transformation of the raw data, or image domain. The later group includes methods that reconstruct images from each coil element with reduced FOV and then merge the images using knowledge of individual coil sensitivities. SenSitivity Encoding (SENSE) [1] is an image domain technique for reconstructing pMRI which requires the sensitivity functions to be given as explicit as possible, thus the sensitivity maps estimation is as important as the reconstruction algorithm.

Both parallel imaging [1-5] and partial Fourier techniques [6-9], which sample only a fraction of the $k$-space data to perform phase-constrained reconstruction [6,7], have been proposed for accelerating data acquisition. 


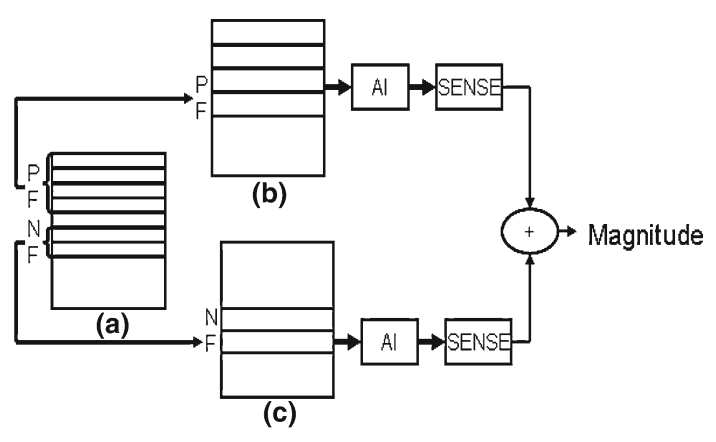

Fig. 1 Analytic image SENSE (AI_SENSE) algorithm. $a-c$ Depicted partial $k$-space data, positive spatial frequencies $(P F)$ and negative spatial frequencies $(N F)$, respectively. AI represents the Analytic Image algorithm

In view of the independence of the two approaches, combining partial $k$-space and pMRI should allow further reductions of the data acquisition time [10-18]. In fact, recent studies have already demonstrated feasibility of this concept such as techniques combining SENSE and partial Fourier [10-14] and homodyne detection SENSE (HM_SENSE) $[11,12]$, which has been claimed to constitute a robust algorithm. An important aspect of these methods is the estimation of the underlying image phase, which is required for improving the SENSE reconstruction also with regard to the acceleration factor. Generally, it can be assumed that the phase of an image is slowly varying in space. However, in case of abrupt phase transitions the phase constraint condition is violated and large reconstruction errors may occur. Phase constrained reconstruction suffers from a lack of high spatial frequencies regarding the image phase and consequently may suffer from residual aliasing in these regions.

In the following, we describe a novel approach of combining pMRI and partial $k$-space techniques that enables overcoming limitations regarding phase estimation by considering the desired image being analytic, meaning that its imaginary and real parts form a Hilbert pair. The method uses the benefits of the analytic image concept $[8,9]$, which exploits data redundancies in $k$-space, for reconstructing the aliased images, which in a second step will be processed by SENSE reconstruction. The analytic image SENSE (AI_SENSE) algorithm illustrated in Fig. 1 allows for reconstructing images of comparable quality to the full $k$-space reconstructed images and considerably decreases the reconstruction error values compared to HM_SENSE reconstruction. It is anticipated that the proposed method can be used for applications of MRI, in which only the magnitude data are needed. We present in the following the analytic image reconstruction combined to the SENSE framework and performance results for in vivo measurements.

\section{Theory}

Analytic image reconstruction

Analytic image reconstruction has been described earlier [8, 9]; for simplicity, we will illustrate the principles as far as they are required for the study presented.

Any given full $k$-space data set $S(u, v)$ can be expressed as the superposition of its negative and positive spatial frequencies in the phase encoding direction, i.e. $S(u, v)=S_{n}(u, v)+$ $S_{p}(u, v)$. Inverse Fourier transformation of $S_{n, p}(u, v)$ yields the real images $f_{n, p}(x, y)$, with $n, p$ standing for negative and positive frequencies. As the Hermitian property is generally not fulfilled for a full $k$-space expressed in this manner, analytic images $z_{p}(x, y)$ and $z_{n}(x, y)$ associated to the positive (or negative) half spatial frequencies were introduced as

$z_{n, p}(x, y)=f_{n, p}(x, y)+\frac{j}{\pi} \cdot p \cdot v \cdot \int_{-\infty}^{+\infty} \frac{f_{n, p}(\tau, y)}{x-\tau} \mathrm{d} \tau$

where $p . v$. stands for the Cauchy principal value, $f_{n, p}(x, y)$ $=\operatorname{Re}\left[F_{2}^{-1}\left(\varphi_{n, p}(u, v)\right)\right]$ with

$\varphi_{n, p}(u, v)=(1+\operatorname{sgn}(u)) F_{2}\left(f_{n, p}\right)(u, v)$

and $F_{2}$ representing the two-dimensional Fourier transform operator. Finally, the analytic image $Z(x, y)$ associated to the full $k$-space is given by the expression:

$$
\begin{aligned}
& Z(x, y)=f_{p}(x, y)+f_{n}(x, y) \\
& +\frac{j}{\pi} p \cdot v \cdot \int_{-\infty}^{+\infty} \frac{f_{p}(\tau, y)}{x-\tau} d \tau+\frac{j}{\pi} p \cdot v \cdot \int_{-\infty}^{+\infty} \frac{f_{n}(\tau, y)}{x-\tau} \mathrm{d} \tau .
\end{aligned}
$$

Assuming now the $k$-space to be sampled asymmetrically (Fig. 1a) comprising all the positive $k$-space frequencies together with few negative frequencies in the phase-encoding direction, Eq. 3 can be rewritten as

$$
\begin{gathered}
Z(x, y)=f_{p}(x, y)+f_{n}(x, y)+\frac{j}{\pi} \cdot p \cdot v \cdot \int_{-\infty}^{+\infty} \frac{f_{p}(\tau, y)}{x-\tau} \mathrm{d} \tau \\
+\frac{j}{\pi} \cdot p \cdot v \cdot \int_{-\infty}^{+\infty} \frac{f_{n}(\tau, y)}{x-\tau} \mathrm{d} \tau \otimes\left(\frac{\delta\left(y_{s}\right)}{2}+\frac{1}{j \cdot 2 \pi \cdot y_{s}}\right),
\end{gathered}
$$

where the last term of the right side of Eq. 4 stands for the Fourier transform of a step function at $y_{s}$ and accounts for the fact that the negative $k$-space is only sampled in part. The final 
reconstructed image $f(x, y)$ is then obtained by computing the magnitude of $Z(x, y)$.

\section{AI_SENSE}

Analytic image reconstruction combined to SENSE (AI SENSE) algorithm aims to reduce reconstruction errors by avoiding the phase estimation step required for phased-constrained SENSE [11,12]. The SENSE algorithm is dependent on the accuracy of the sensitivity maps estimation, which may not be straightforward. Poor sensitivity map estimates may lead to poor convergence of the algorithm and produce image artifacts. Phase-constrained SENSE reconstructions may account for part of these deficiencies, yet also in this case deviations from the true image phase occur.

Using the analytic image reconstruction algorithm, one can largely avoid the phase estimation step. For SENSE reconstruction, we express the analytic image as a linear combination of the individual coil contributions

$z_{n, p}=C \rho_{n, p}+\varepsilon_{n, p}$

with $\mathbf{C}$ being a $\left(N_{k} N_{c}\right) \times N^{2}$ complex matrix representing the coil sensitivities and Fourier kernel for an image of $N \times N$ pixels, and $\varepsilon$ a $\left(N_{k} N_{c}\right) \times 1$ complex vector representing independent measurement noise, and $\rho_{n, p}$ represents the unaliased complex magnetization for a set of $N^{2}$ pixels.

SENSE reconstruction is then carried out according to published procedures [1], i.e.

$\mathrm{z}^{\mathrm{c}}=\left(\mathrm{C}^{\mathrm{H}} \psi^{-1}\right)^{-1} \mathrm{C}^{\mathrm{H}} \psi^{-1} \mathrm{Z}$

with $\psi=\Psi n+\Psi p$ being the noise covariance matrix and $\mathrm{Z}$ the term equal to $z_{n}+z_{p}$ (eq. 4 ).

$\mathbf{z}^{C}$ stands for the complex reconstruction estimated in a minimum least-squares sense and which magnitude represents the desired image.

The AI_SENSE algorithm illustrated in Fig. 1 mostly differs from the HM_SENSE approach by the fact that it skips the step of computing a reference of the phase distribution throughout the image. Two aliased analytic images are reconstructed using the negative and the positive spatial frequencies, which are then processed through SENSE algorithm for unwrapping. The final reconstructed image is then obtained as magnitude of the sum of the two SENSE-like reconstructed images (Eq. 6).

To improve the reconstruction quality of HM_SENSE, an additional intensity constraint was incorporated in its algorithm. An intensity threshold $\mathrm{V}$ has been defined in the reconstructed image and values lower than $\mathrm{V}$ were set to zero.

$I_{R_{V}}(X)= \begin{cases}I_{R}(X) & |X|>V \\ 0 & \text { otherwise }\end{cases}$ with $I_{R_{V}}$ representing the HM_SENSE_C reconstructed image. This allowed reducing the error in the HM_SENSE reconstructed images.

\section{Materials and methods}

The AI-SENSE reconstruction method was implemented in Matlab (R2007b, MathWorks, Inc, USA). To test its performance, in vivo experiments were achieved on healthy Lewis rats. All the shown data sets were acquired on a Bruker BioSpec 94/30 (Bruker BioSpin MRI, Ettlingen, Germany) using a four-element phased array surface coil for reception and a linear polarized volume resonator coil for excitation. For comparison with HM_SENSE, both spin echo and gradient echo based sequences were applied in order to evaluate the performance of the two reconstruction methods in case of deviations from the Hermitian symmetry due to local phase variations.

\section{MR experiments}

In vivo experiments were carried out on male Lewis rats and in strict adherence to the Swiss law for animal protection.

For computing sensitivity maps, low resolution images were acquired with the volume resonator and with each of the four element phased array coil. 2D Rapid Acquisition with Relaxation Enhancement (RARE) pulse sequence has been used with the following parameters: TE/TR $=15 / 2,000 \mathrm{~ms}$, $\mathrm{FOV}=4 \times 3 \mathrm{~cm}$, matrix $=128 \times 16$, Slice thickness $=$ $1 \mathrm{~mm}$ with a RARE factor $=1$, was used. Sensitivity maps $S_{C i}(x, y)$ were obtained as ratios of individual coil images $I_{C i}(x, y)$ to the volume resonator $I_{V B}(x, y)$

$S_{C i}(x, y)=\frac{I_{C i}(x, y)}{I_{V R}(x, y)}$

The computed sensitivity maps were then thresholded using a mask having the following expression

$\operatorname{Mask}(x, y)= \begin{cases}1 & \text { for } I_{W B}(x, y) \geq I_{t h} \\ 0 & \text { otherwise }\end{cases}$

Equation 9 represents the set of images with a limited object support. The values outside the support were considered noise and set to zero whereas the values inside were set to the 1 .

Following the acquisition of low resolution data sets for sensitivity maps estimation, full resolution array coil data sets were acquired using the sequence parameters presented above with the difference of filling a $128 \times 128$ matrix size. Every alternate phase encoding line was then manually removed to simulate undersampling in pMRI. The full $k$-space data of all the involved coils were combined to obtain the Roemer [19] reconstruction, which serves as reference 
for comparison. The subsampled data sets with different reduction factor were used to reconstruct the desired images through the proposed AI_SENSE as well as the HM_SENSE techniques.

$\mathrm{T}_{2}^{*}$-weighted images were acquired using a 2D Fast Low Angle Shot (FLASH) pulse sequence with the following acquisition parameters: TE/TR $=4.468 / 500 \mathrm{~ms}, \mathrm{FOV}=3$ $\times 3 \mathrm{~cm}$, matrix $=256 \times 256$, Slice thickness $=1 \mathrm{~mm}$, flip angle $=30^{\circ}$. The sensitivity maps were estimated using Eq. 10

$\operatorname{SE}_{C i}(x, y)=\frac{I_{C i}(x, y)}{\sqrt{\sum_{i=1}^{N_{C}}\left|I_{C i}(x, y)\right|^{2} / N_{C}}}$

with $I_{C i}(x, y)$ being the image acquired with each single coil and $N_{C}$ the number of coils. The $S E_{C i}(x, y)$ maps were then masked as described above. The data set was acquired covering full $k$-space; undersampling of pMRI was mimicked by omitting phase encoding lines according to the chosen acceleration factor. The full $k$-space data of all the involved coils were combined using the Roemer reconstruction to generate an image taken as reference in this anatomical comparison.

Image analysis

\section{Performance analysis}

The performance of the various reconstruction methods (HM_SENSE,HM_SENSE_C and AI_SENSE) was visually evaluated in terms of image quality and by analyzing difference images between the reference and accelerated $k$-space reconstructed images.

Reconstructions were also quantitatively compared in terms of normalized mean square error (NMSE) defined as

$\mathrm{NMSE}=\frac{1}{N} \sum \sum \frac{\left(I_{\mathrm{ref}}(x, y)-I_{\mathrm{acc}}(x, y)\right)^{2}}{\bar{I}_{\mathrm{ref}}(x, y) \cdot \bar{I}_{\mathrm{acc}}(x, y)}$,

where $I_{\mathrm{ref}}(x, y)$ is the reference and $I_{\mathrm{acc}}(x, y)$ the $\mathrm{HM}_{-}$ SENSE,HM_SENSE_C or AI_SENSE reconstructed image. $\bar{I}_{\text {ref }}(x, y)$ and $\bar{I}_{\text {acc }}(x, y)$ are the mean image intensities of $I_{\text {ref }}(x, y)$ and $I_{\text {acc }}(x, y)$, respectively.

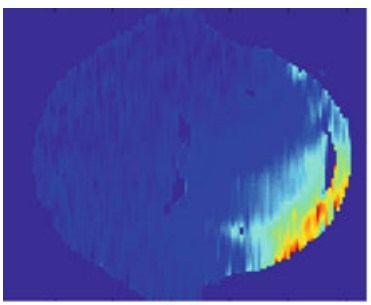

(a)

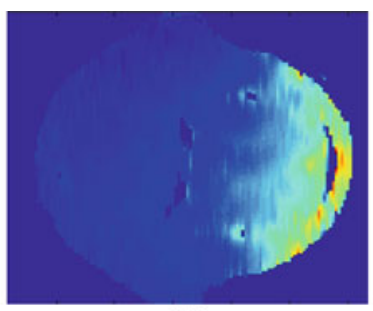

(b)

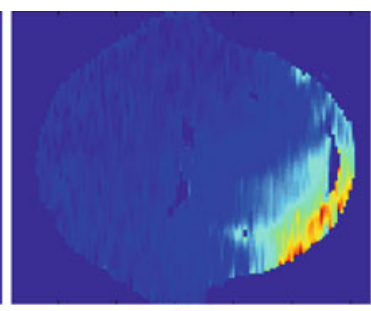

(c)

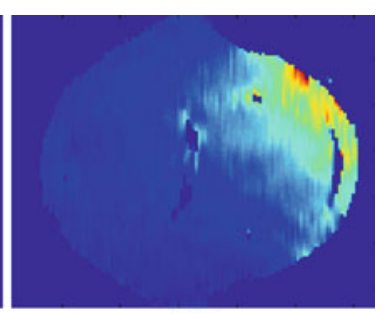

(d)

Fig. 2 Estimated sensitivity maps of the four channel phased array after interpolation, thresholding, and filtering. a-d First to fourth channel 


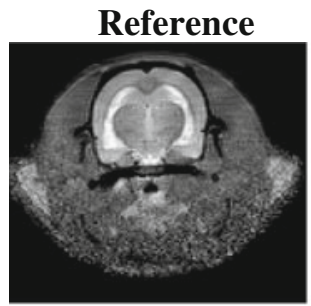

(a)

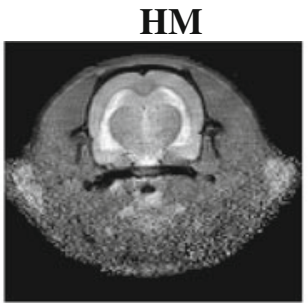

(b)

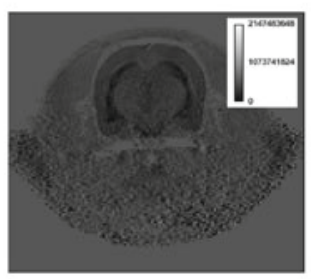

(e) $\mathrm{AP}=0.085 ;$ Nmse $=0.0414$

$\mathrm{PF}=1.6$

$\mathrm{R}=3.2$

$R=4$

$R=6.4$

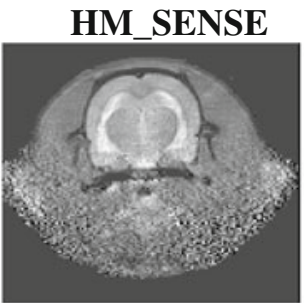

(h)

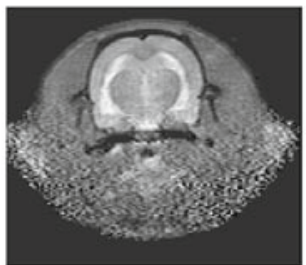

(k)

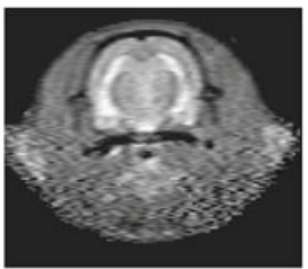

(n)

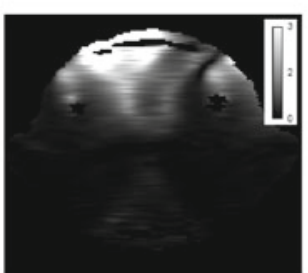

(q)

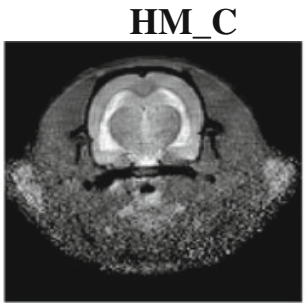

(c)

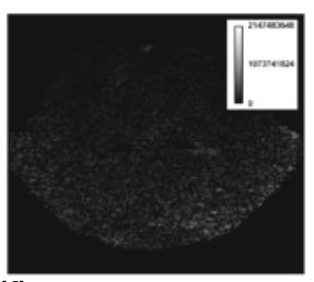

(f) $\mathrm{AP}=0.0026 ;$ Nmse $=0.0026$

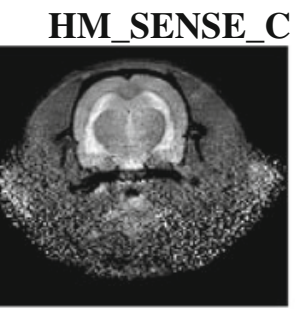

(i)

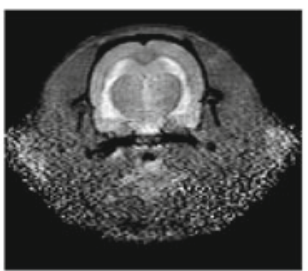

(I)

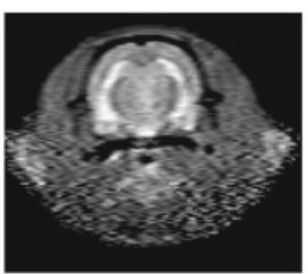

(o)

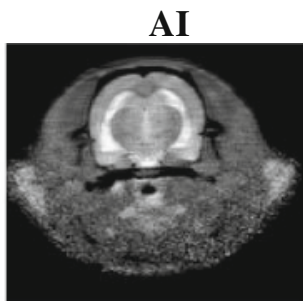

(d)

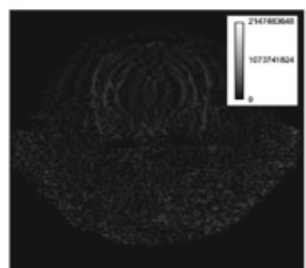

(g) $\mathrm{AP}=0.007 ;$ Nmse $=0.007$

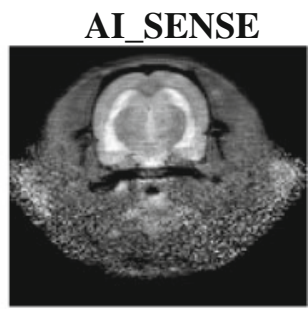

(j)

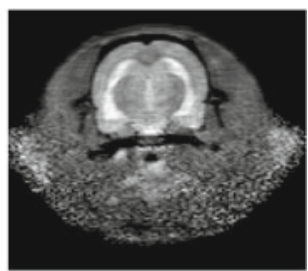

(m)

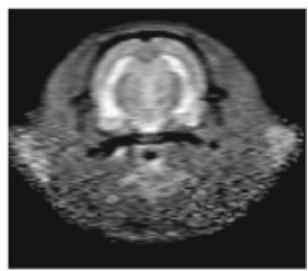

(p)

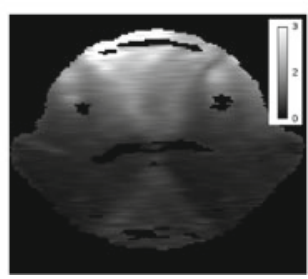

(r)

Fig. 3 Reconstruction results from computer simulation on a rat brain data set. The first row represents a reference image reconstructed with full $k$-space, b-d are images reconstructed with conventional homodyne detection (HM), constrained HM (HM_C) and analytic image $(A I)$ algorithms, without undersampling and with a reduction factor of 1.6, respectively. NMSE stands for the error values and AP for the artefact power values. The second row represents images combining pMRI $(\mathrm{R} 1=2)$ and partial $k$-space acquisition (reduction factor $(\mathrm{RF})=1.6)$

leading to a final acceleration factor of 3.2. $\mathbf{h}-\mathbf{j}$ are images reconstructed with HM-SENSE, HM-SENSE_C and AI-SENSE, respectively. To further increase the reduction factor for partial $k$-space, part of positive high spatial frequencies are not acquired and lead to different acceleration factors. $\mathbf{k}-\mathbf{m} \mathrm{R}=4(\mathrm{R} 1=2, \mathrm{RF}=2)$ and $\mathbf{n}-\mathbf{p} \mathrm{R}=6.4(\mathrm{R} 1=2, \mathrm{RF}$ $=3.2$ ). The g-maps are also shown on the bottom row corresponding to the sensitivities from $\mathbf{m}$ HM_SENSE and $\mathbf{n}$ AI_SENSE 
Table 1 Simulation of image acquisition by combining partial $k$-space with parallel acquisition using 4 receiver coils

\begin{tabular}{lllllll}
\hline Images & $R F$ & $R 1$ & $N_{n}$ & $N_{p}$ & Number of $k$-space lines & Acceleration PF. R1 \\
\hline (b)-(d) & 1.6 & 1 & 16 & 64 & 80 & 1.6 \\
(h)-(j) & 1.6 & 2 & 8 & 32 & 40 & 3.2 \\
(k)-(n) & 2.0 & 2 & 8 & 24 & 32 & 4.0 \\
(o)-(q) & 3.2 & 2 & 8 & 12 & 20 & 6.4 \\
\hline
\end{tabular}

Acceleration factors are indicated as $P F$ and $R$, respectively. $N_{p, n}$ indicates the number of $k$-space lines sampled for negative and positive frequencies starting from the center of $k$-space

Fig. 4 Quantitative assessments: a, b: AP, SNR as a respectively. The SNR values homogeneous ROI of each image (illustration on the figure) function of acceleration factor, were measured in a
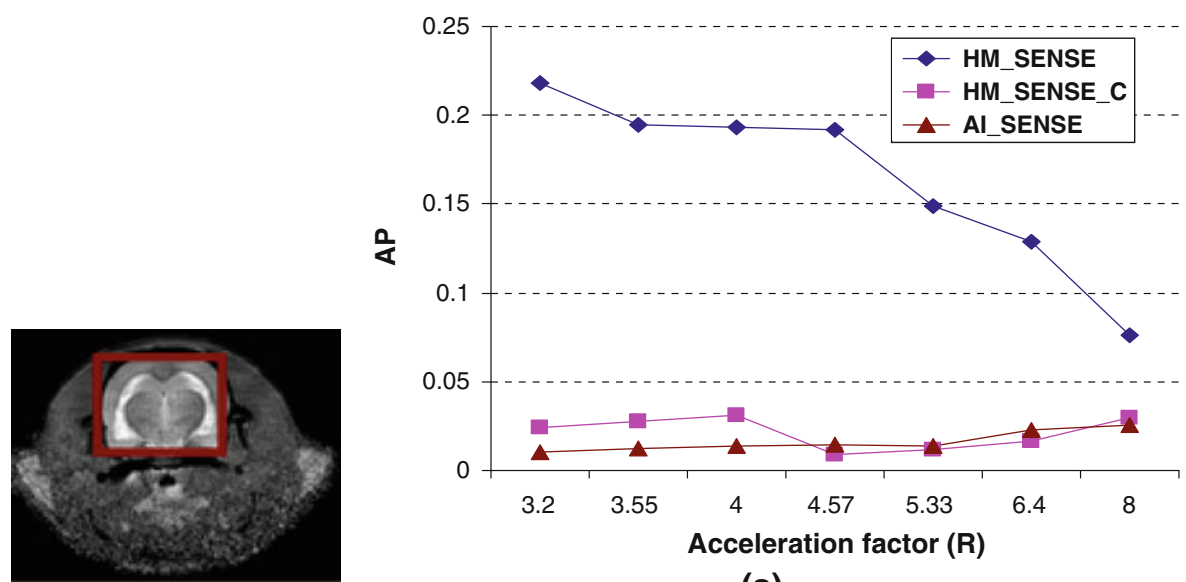

(a)
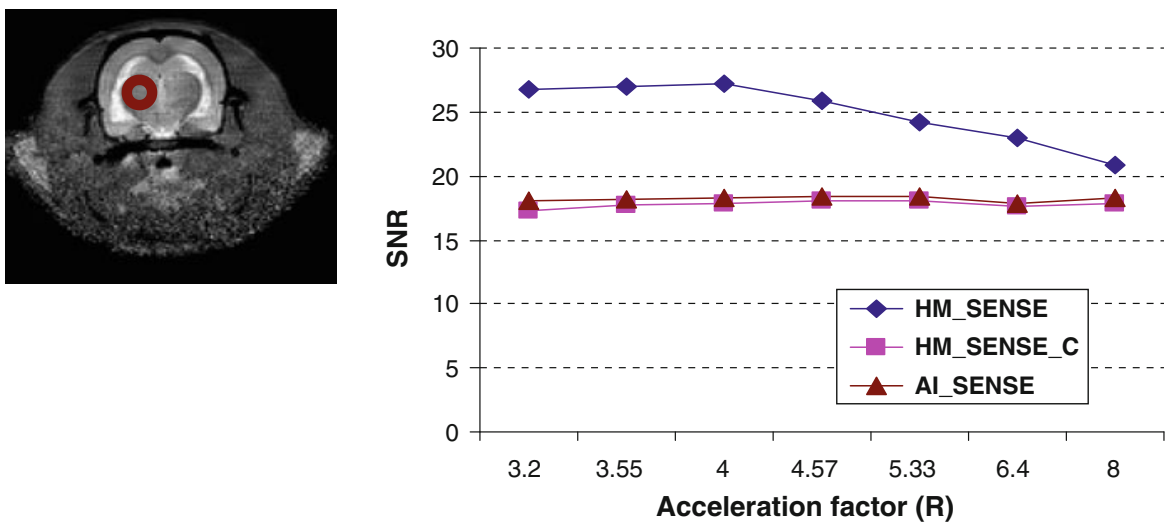

(b)

computed and shown in Fig. 3q, r, but they naturally do not provide information about noise amplification. All images were normalized and shown at the same intensity level. The algorithm accuracy was evaluated in terms of NMSE and artifact power (AP). It can be seen that AI_SENSE provides superior image quality than HM_SENSE even when its intensity values are constrained in order to improve the reconstruction quality (HM_SENSE_C). Visual analyses as well as the quantitative assessments well depict this superiority. Difference images (data not shown), revealed that HM_SENSE reconstruction error manifest themselves throughout the image, while regions displaying significant errors in AI_SENSE reconstruction are mostly confined to regions showing sharp intensity transitions, which can easily be explained by the lack of a part of the high spatial frequencies.

Figure 4a plots artifact power values (AP) and signal to noise ratio values (SNR) as functions of the total acceleration factor, respectively. These values were computed in central region-of-interest within the brain (see insert) to avoid interference by the background signal encountered in HM_SENSE, which was not of interest in this study. Based on the error metric, AP, AI_SENSE and HM_SENSE_C are clearly superior to HM_SENSE, while the latter method displays better sensitivity as reflected by the higher SNR values. Interestingly, for high combined acceleration values, 
Fig. 5 The left column illustrates the reconstructed brain images from a set of 4-channel a full $k$-space data using ROEMER reconstruction and subsampled data using b HM_SENSE,

c HM_SENSE_C and d AI_SENSE. The difference images between e ROEMER and HM_SENSE, $\mathbf{f}$ ROEMER and HM_SENSE_C and g ROEMER and AI_SENSE

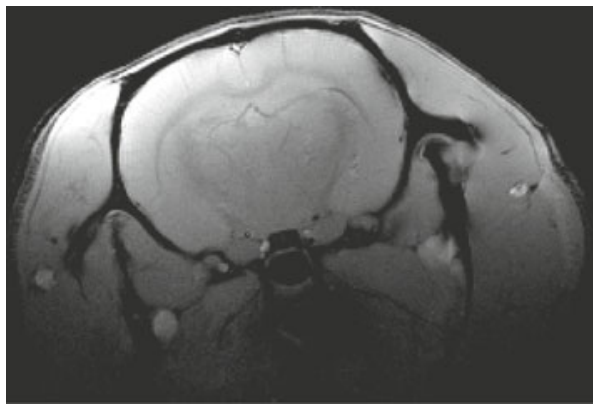

(a)

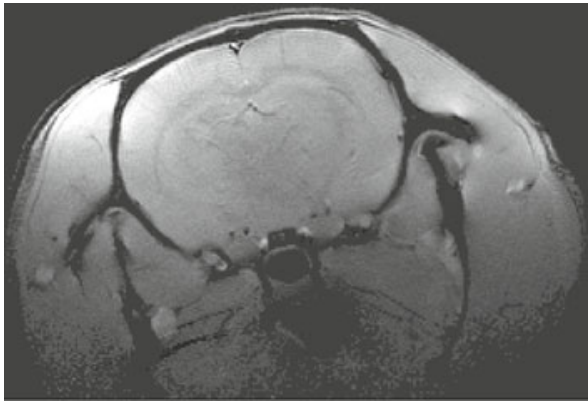

(b)

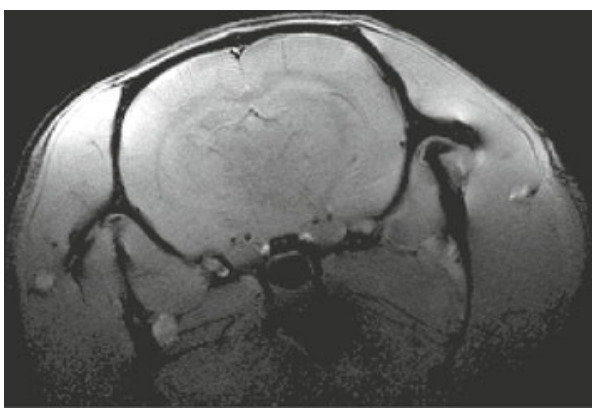

(c)

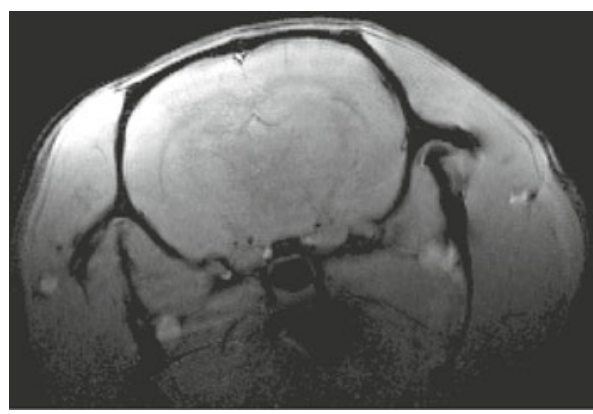

(d)

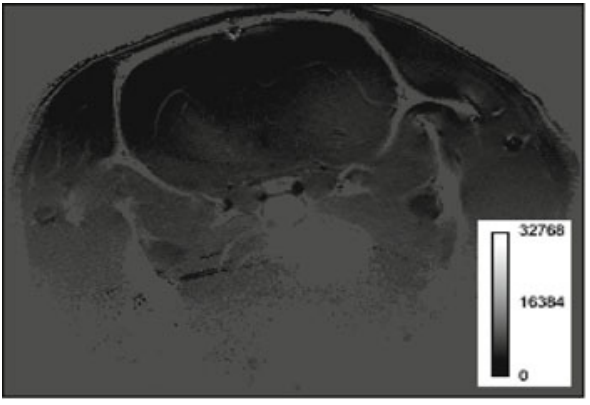

(e) $\mathrm{NMSE}=0.167$

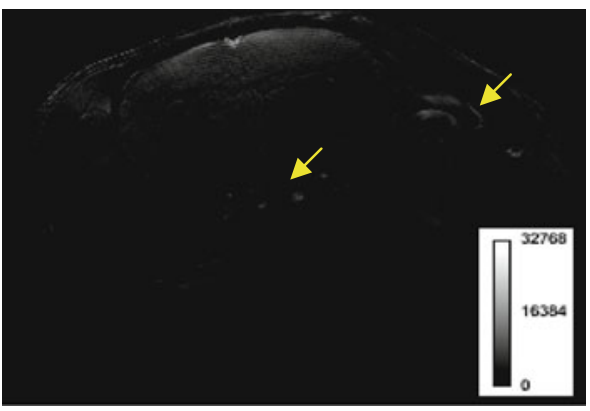

(f) $\mathrm{NMSE}=0.008$

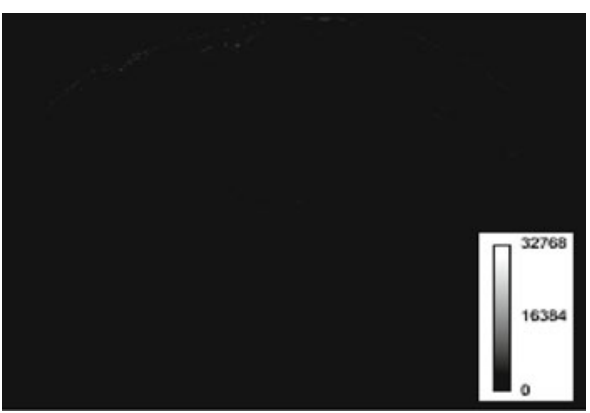

(g) NMSE $=0.004$ the error values of all three approaches become similar. The error values curve plotted for HM_SENSE, data not shown, does not describe the expected behavior of the error evolution when the number of phase encoding lines, used for reconstruction, is reduced. This is probably due to the noise level in the reconstructed image. The noise level is drastically reduced as the amount of positive high spatial frequencies is decreased, leading to a decrease of the error values. The AP values consolidate this statement as we know that its values could be elevated due to noise even when the folding artifacts are completely removed.

Figure 5 illustrates reconstruction results of in vivo rat brain images from an undersampled $k$-space data set $(R=$ 3.2) acquired with a FLASH sequence. A high resolution image was acquired prior to the undersampled $k$-space data sets used for reconstruction. Central symmetric $32 k$-space 
lines were used for sensitivity maps estimation while the full $k$-space data was used for reconstructing reference image. The images reconstructed from undersampled data reveal that different approaches discussed lead to satisfactory image reconstructions. Nevertheless, the results obtained by using AI_SENSE appear slightly superior as concluded from both visual comparisons and difference images. HM_SENSE_C underestimates intensity values in regions displaying high changes in signal intensity, in particular at edges (see arrows on Fig. 5f). This reflected the lack of high frequency components in the data used for phase maps estimation. On the other hand HM_SENSE amplifies the noise level during the reconstruction process while having less variation between intensity values, which explains high error values computed with HM_SENSE. The difference images depicted in Fig. 3e-g well illustrate the statement. The results also show that AI_SENSE is less sensitive to errors in sensitivity estimation than HM_SENSE. In order to avoid any intensity attenuations, we did not use any smoothing function in the AI_SENSE algorithm which explains some ringing artifacts observed in AI_SENSE reconstructed images (Fig. 5g).

\section{Discussion}

In this study, we have introduced the AI_SENSE concept combining pMRI with partial $k$-space sampling and the results demonstrate that AI_SENSE processing allows for further acceleration of the data acquisition of conventional SENSE with little impact on the quality of the reconstructed images. The analytic image concept has already been used to reconstruct partial $k$-space data both from static [8] and dynamic [9] objects. In this work we demonstrated that the combination of this concept to SENSE successfully and robustly reconstructs images while achieving higher acceleration factors. This technique reconstructs undersampled $k$-space data from multiple coils using the analytic image concept yielding aliased images which are then unwrapped through SENSE processing. In particular, the approach proposed does not require any phase correction in its algorithm, which constitutes a prominent source of reconstruction errors (Fig. 5). As the phase maps are commonly estimated from low resolution images, sometimes even Hamming filtered, residual phase error will emerge due to lack of high spatial frequency contributions. We also observed that AI_SENSE provides superior results both in terms of qualitative and quantitative assessments when compared to HM_SENSE. HM_SENSE yields higher SNR values than AI_SENSE despite unitary g-factors. Another explanation could be that HM_SENSE eliminates the imaginary channel noise which results in approximately a $\sqrt{2}$ reduction in noise as compared with an AI_SENSE magnitude image containing noise from both real and imaginary parts.
For modest or high acceleration factors, AI_SENSE seems to be accurate even using low resolution images for sensitivity maps estimation compare to HM_SENSE which starts diverging at high acceleration factors.

\section{Conclusions}

Combining pMRI with partial $k$-space sampling allows accelerating data acquisition beyond conventional pMRI using e.g. SENSE. Using an appropriate image reconstruction algorithm such as AI_SENSE largely preserves the image quality. In this regard AI_SENSE outperforms HM_SENSE. Even for high acceleration factors $\left(R \geq N_{c}\right)$ images of good quality with minor reconstruction errors are obtained. The method is therefore attractive for experiments requiring fast data acquisition e.g. 3D dynamic imaging. Collecting images at a higher rate will improve the quality of temporal fitting procedures and may reduce the propensity of motion artifacts.

Acknowledgments We thank the Swiss National Science Foundation for funding.

Open Access This article is distributed under the terms of the Creative Commons Attribution Noncommercial License which permits any noncommercial use, distribution, and reproduction in any medium, provided the original author(s) and source are credited.

\section{References}

1. Pruessmann KP, Weiger M, Scheidegger MB, Boesiger $P$ (1999) Sensitivity encoding for fast MRI. Magn Reson Med 42:952-962

2. Griswold MA, Jakob PM, Heidemann RM, Nitka M, Jellus V, Wang J, Kiefer B, Haase A (2002) Generalized autocalibrating partially parallel acquisitions (GRAPPA). Magn Reson Med 47: $1202-1210$

3. Griswold MA, Jakob PM, Nitka M, Goldfarb JW, Haase A (2000) Partially parallel imaging with localized sensitivities (PILS). Magn Reson Med 44:602-609

4. Sodickson DK, Manning WJ (1997) Simultaneous acquisition of spatial harmonics (SMASH): fast imaging with radiofrequency coil array. Magn Reson Med 38:591-603

5. Heidemann RM, Griswold MA, Haase A, Jakob PM (2001) VD-AUTO-SMASH imaging. Magn Reson Med 45:1066-1074

6. Noll DC, Nishimura DG, Macovski A (1991) Homodyne detection in magnetic resonance imaging. IEEE Trans Med Imaging 10: 63-154

7. McGibney G, Smith MR, Nicholas ST, Crawley A (1993) Quantitative evaluation of several partial-Fourier reconstruction algorithms used in MRI. Magn Reson Med 30:51-59

8. Yankam Njiwa J, Zhu YM, Robini Marc, Magnin I (2007) Magnetic resonance image reconstruction using the notion of analytic image. Nucl Instr Meth Phys Res A 1-2:73-76

9. Yankam Njiwa J, Hiba B, Zhu YM (2007) Cardiac Cine MR reconstruction from partial $\mathrm{k}$-space using the notion of analytic image. In: IEEE EMBC conference, Lyon, France, pp 2057-2060 
10. Madore B (2002) Using UNFOLD to remove artifacts in parallel imaging and in partial-fourier imaging. Magn Reson Med 48: 493-501

11. King et al (2000) Proceedings of Soc. Magnetic Resonance Medicine, vol 8, p 153

12. Willig-Onwuachi JD, Yeh EN, Grantr AK, Ohliger MA, McKenzie CA, Sodickson DK (2005) Phase-constrained parallel MR image reconstrcution. JMR 176:187-198

13. Samsonov AA, Kholmovski EG, Parker DL, Johnson CR (2004) POCSENSE: POCS-based reconstruction for sensitivity encoded magnitic resonance imaging. Magn Reson Med 52:1397-1402

14. Lew C, Pineda AR, Clayton D, Spielman D, Chan F, Bammer R (2007) SENSE phased-constrained magnitude reconstruction with iterative phase refinement. Magn Reson Med 58:910-921
15. Bydder M, Robson MD (2005) Partial Fourier partially parallel imaging. Magn Reson Med 53:1393-1401

16. Ying L, Sheng J (2007) Joint image reconstruction and sensitivity estimation in SENSE (JSENSE). Magn Reson Med 57:1196-1202

17. Kellman P, Epstein FH, McVeigh ER, Crawley A (2001) Adaptative sensitivity encoding incorporating temporal filtering (TSENSE). Magn Reson Med 45:846-852

18. Madore B (2004) UNFOLD-SENSE: a parallel MRI method with self-calibration and artifacts suppression. Magn Reson Med 52:310-320

19. Roemer PB, Edelstein WA, Hayes CE, Souza SP, Muelle OM (1990) The NMR phased array. Magn Reson Med 16:192-225 